\title{
Review Article \\ Natural Products as Adjunctive Treatment for Pancreatic Cancer: Recent Trends and Advancements
}

\author{
Qingxi Yue, ${ }^{1,2}$ Guogang Gao, ${ }^{3}$ Gangyong Zou, ${ }^{4}$ Haiqing Yu, ${ }^{5}$ and Xi Zheng ${ }^{2,6}$ \\ ${ }^{1}$ Department of Oncology, Shanghai 9th People's Hospital, Shanghai Jiao Tong University School of Medicine, Shanghai, China \\ ${ }^{2}$ Department of Chemical Biology, Ernest Mario School of Pharmacy, Rutgers, The State University of New Jersey, Piscataway, NJ, USA \\ ${ }^{3}$ Department of Cardiothoracic Surgery, Weihai Municipal Hospital, Weihai, Shandong, China \\ ${ }^{4}$ Department of Pathology, Weihai Municipal Hospital, Weihai, Shandong, China \\ ${ }^{5}$ Department of Internal Medicine, University of Missouri Hospital and Clinics, Columbia, MO, USA \\ ${ }^{6}$ Allan H. Conney Laboratory for Anticancer Research, School of Chemical Engineering and Light Industry, \\ Guangdong University of Technology, Guangzhou, China
}

Correspondence should be addressed to Qingxi Yue; qxyue@sibs.ac.cn and Xi Zheng; xizheng@pharmacy.rutgers.edu

Received 3 August 2016; Revised 9 December 2016; Accepted 27 December 2016; Published 23 January 2017

Academic Editor: Li Jiao

Copyright (C) 2017 Qingxi Yue et al. This is an open access article distributed under the Creative Commons Attribution License, which permits unrestricted use, distribution, and reproduction in any medium, provided the original work is properly cited.

Pancreatic cancer is a type of common malignant tumors with high occurrence in the world. Most patients presented in clinic had pancreatic cancer at advanced stages. Furthermore, chemotherapy or radiotherapy had very limited success in treating pancreatic cancer. Complementary and alternative medicines, such as natural products/herbal medicines, represent exciting adjunctive therapies. In this review, we summarize the recent advances of using natural products/herbal medicines, such as Chinese herbal medicine, in combination with conventional chemotherapeutic agents to treat pancreatic cancer in preclinical and clinical trials.

\section{Introduction}

Pancreatic cancer is a type of common malignant tumors with high occurrence in the world. Due to the high rate of invasion and malignancy and asymptomatic development, pancreatic cancer is highly lethal. Data from Global Cancer Statistics in 2012 indicated that pancreatic cancer was the seventh leading death cause from cancer in both men and women worldwide [1]. In the developed countries, pancreatic cancer was the fifth/fourth leading death cause from cancer in men and women, respectively. In the developing countries, pancreatic cancer was the eighth/tenth leading death cause from cancer in men and women, respectively [1]. The patients with pancreatic cancer had poor survival rate with less than $5 \%$ of patients surviving 5 years after diagnosis. In 2016, it was estimated that 53,070 patients will be diagnosed with pancreatic cancer and 41,780 patients with pancreatic cancer will die in the United States, most of them dying within first year of diagnosis [2]. According to the 2015 oncology annals, pancreatic cancer in China was the ninth/sixth leading death cause from cancer for men and woman, respectively. About
90,100 patients were diagnosed with pancreatic cancer and 79,400 patients in China with pancreatic cancer died in 2015 [3]. Despite recent improvements of diagnostic techniques, most pancreatic cancer patients were diagnosed at advanced states. Most patients with pancreatic cancer, who were diagnosed annually, died within a year of diagnosis. So far, there are no adequate therapies for treating the patients with pancreatic cancer.

Surgery is a pivotal curative therapeutic approach for most patients with pancreatic cancer. However, the success rate of resection surgery remains very low because about $80 \%-85 \%$ patients with diagnosed with pancreatic cancer were already in an advanced stage [4]. So, only $15 \%-20 \%$ of patients with pancreatic cancer are eligible for surgical resection after being primarily diagnosed. Standard surgical procedures include pancreaticoduodenectomy (pyloruspreserving or stomach-preserving) for tumors of pancreatic head and distal pancreatectomy with splenectomy for tumors arising in the tail or body of the pancreas [4]. Radical resection, alone or in combination with other therapy, is the only way to eradicate pancreatic cancer. Only $10 \%-20 \%$ of 
patients with pancreatic cancer, who have radical resection, can survive 5 years. So, it is necessary to underscore the need for better preoperative staging and more effective systemic therapy [5]. Therefore, chemotherapy and radiotherapy are considered as the standard treatment approaches for the patients with unresectable pancreatic cancer, especially for locally advanced or metastatic inoperable patients with pancreatic cancer.

In the past three decades, the standard therapeutic drugs for pancreatic cancer were fluoropyrimidine drug 5fluorouracil (5-FU) and the antimetabolite drug gemcitabine [6]. Since 5-fluorouracil (5-FU) was generated 50 years ago, only the incremental changes in clinical outcomes of pancreatic cancers were made. 5-Fluorouracil (5-FU) was the first agent to be widely used to treat advanced pancreatic cancer, but the success rates of 5-FU were $<20 \%$ and it was not known whether 5-FU could provide significant palliative benefits. Gemcitabine, which is now widely accepted as the golden-standard drug prescribed for the patients suffering from locally advanced (stage II or stage III) or metastatic (stage IV) pancreatic cancer, is an analog of the pyrimidine nucleotide deoxycytidine [7]. Although gemcitabine offered only an extension of $\sim 1.5$ months in median survival, gemcitabine replaced 5-fluorouracil (5-FU) as the standard firstline chemotherapeutic agent since it was approved by the Food and Drug Administration (FDA) in 1996 [7]. Current treatment modalities for the advanced pancreatic cancer include gemcitabine, as a single agent or in combination with multiple chemotherapeutic agents. Unfortunately, most patients with locally advanced and metastatic pancreatic cancers could not benefit from the monotherapy of gemcitabine. Though many clinical trials had been conducted to ascertain the optimum therapy utilizing gemcitabine in combination with multiple chemotherapeutic agents such as 5-FU, capecitabine, pemetrexed, topoisomerase inhibitors, epidermal growth factor receptor (EGFR) tyrosine kinase inhibitors, erlotinib, bevacizumab, irinotecan, exatecan, platinum compounds (cisplatin and oxaliplatin), and taxanes (paclitaxel and docetaxel), almost none of them was proven to be more effective, in comparison with gemcitabine as a single agent for treating pancreatic cancer [8]. In 2005, a four-drug regimen (gemcitabine, 5-fluorouracil, epirubicin, and cisplatin) was shown to improve the progression free survival and the overall survival of the patients with pancreatic cancer, compared with single-agent gemcitabine [8]. In 2011, a new treatment regimen FOLFIRINOX (a combination of 5-FU, leucovorin/folinic acid, oxaliplatin, and irinotecan) was demonstrated as superior survival outcomes of the patients with pancreatic cancer, compared with single-agent gemcitabine, which led to the adoption of FOLFIRINOX as the preferred option for the patients [8]. A trial comparing gemcitabine/nab-paclitaxel (nanoparticle albumin-bound paclitaxel) with gemcitabine alone showed a statistically significant survival benefit for the new doublet to introduce another option for the treatment of advanced pancreatic cancer [8].

Radiotherapy is often used to combine with systemic chemotherapy in oncology centers of USA. Radiotherapy exhibits a substantial advantage with respect to local control and improving the resectability rate after downstaging. Nonetheless, the approach has not significantly improved survival rates of the patients with unresectable pancreatic cancer. Moreover, specific radiotherapy modalities, such as stereotactic radiotherapy, TOMO, and intensity-modulated radiotherapy, have been applied to treat pancreatic cancer and survival outcomes of the patients with pancreatic cancer are partially improved [9]. However, compared with tumors in other sites, the overall survival of the patients with pancreatic cancer is unsatisfactory and the toxicity of radiotherapy is obvious. The poor prognosis of the patients with pancreatic cancer has been attributed to its late diagnosis, limitation of surgical resection, early metastases, aggressive local invasion, and robust resistance to chemotherapy and radiotherapy [9]. Hence, additional therapeutic approaches for treating pancreatic cancer are critical.

Complementary and alternative medicines, such as gene therapy, immunotherapy, targeted therapy, neoadjuvant therapy, and natural products/herbal medicines, may benefit the patients with pancreatic cancer as a supplementary therapy [10]. Of all complementary and alternative medicines, natural products/herbal medicines, such as Chinese herbal medicine, have become popular in the patients with advanced cancer, due to its efficacy and low toxicity [10]. Recent research works indicated that natural products/herbal medicines, such as Chinese herbal medicine, could provide additional strategies for monotherapy or combination treatments of various cancer types, including pancreatic cancer. Indeed, more than $60 \%$ of the current anticancer chemotherapeutic drugs used in clinic were initially developed from natural products/herbal medicines [11]. Natural products/herbal medicines, combined with conventional chemotherapy and radiotherapy, may enhance anticancer therapeutic efficacy and reduce the side effects [12]. In this context, the use of natural products/herbal medicines, as a supplementary approach, to treat pancreatic cancer holds a great promise.

Based on the published papers (up to July 15, 2016) searched in PubMed using the key words "natural product AND combination AND pancreatic cancer," we summarized the beneficial effects of natural products/herbal medicines, such as Chinese herbal medicine, in combination with conventional chemotherapy for the patients with unresectable pancreatic cancer in preclinical and clinical trials in this review. The detailed information of the current review includes (1) combination of natural products with gemcitabine, (2) combination of natural products with other chemotherapeutic agents, (3) combination among natural products, and (4) combination of natural products in early phases of clinical trials.

\section{Combination of Natural Products with Gemcitabine}

Irofulven (MGI 114, HMAF, 6-hydroxymethylacylfulvene), a novel cytotoxic agent synthesized from the sesquiterpene mushroom metabolite of the natural product illudin $\mathrm{S}$, has a unique mechanism of action involving the formation of macromolecule adduct, cell cycle arrest of S-phase, and induction of apoptosis [49]. Phase I clinical combination 
trial study of irofulven and gemcitabine in the patients with advanced solid tumor was underway [50]. Van Laar et al. showed similar marked activity of irofulven against pancreatic xenografts which was observed at lower total doses of an intermittent dosing regimen, compared to consecutive daily dosing [51]. Further, enhanced antitumor activity was observed when irofulven and gemcitabine were combined against the MiaPaCa pancreatic xenograft model, which indicated at least an additive interaction compared to singleagent activity. Psorospermin, a natural product isolated from the stembark and roots of the African plant Psorospermum febrifugum, had the activity against drug-resistant leukemia lines and AIDS-related lymphoma [52, 53]. Fellows et al. showed that psorospermin had the same effect as gemcitabine in inhibiting the growth of tumor in vivo in the MiaPaCa pancreatic xenograft model. Moreover, psorospermin combined with gemcitabine was found to have an at least additive effect in slowing the growth of MiaPaCa pancreatic cancer cells [54]. 3,3-Diindolylmethane (DIM) is a natural compound which can be easily obtained by consuming the cruciferous vegetables. Banerjee et al. present in vitro and in vivo preclinical evidence supporting chemosensitization of pancreatic cancer cells by DIM [13]. DIM potentiates chemosensitization and killing of pancreatic cancer cells by downregulation of constitutive as well as drug-induced activation of NFkappaB and its downstream genes (XIAP, Bcl-xL, survivin, and cIAP). Compared with monotherapy, DIM pretreatment of pancreatic cancer cells resulted in a significantly increased apoptosis with suboptimal concentrations of chemotherapeutic agents such as gemcitabine, cisplatin, and oxaliplatin. Thymoquinone is a bioactive compound extracted from the oil of folklore medicine black seed (Nigella sativa). Banerjee et al. reported the chemosensitizing effect of thymoquinone to conventional chemotherapeutic agents (gemcitabine and oxaliplatin) both in vitro and in vivo using an orthotopic model of pancreatic cancer [14]. By downregulation of nuclear factor-kappaB (NF-kappaB/NF- $\kappa \mathrm{B}), \mathrm{Bcl}-2$ family, and NF-kappaB-dependent antiapoptotic genes (survivin, Xlinked inhibitors of apoptosis, and cyclooxygenase-2), thymoquinone could potentiate the killing of pancreatic cancer cells induced by chemotherapeutic agents (gemcitabine and oxaliplatin). Cucurbitacin B, a member of the cucurbitacins, is derived from Cucurbitaceae family "Trichosanthes kirilowii Maximowicz," a plant that has long been used in oriental medicine for its abortifacient, antidiabetic, and antiinflammatory effects. Thoennissen et al. for the first time showed that cucurbitacin $B$ has profound antiproliferative effects against human pancreatic cancer cells in vitro and in vivo and cucurbitacin B may potentate the antiproliferative activity of nucleoside analogue gemcitabine, associated with inhibition of activated JAK2/STAT3 and decrease of expression of Bcl-XL with subsequent upregulation of caspase3 and caspase-9 [15]. Isothiocyanate sulforaphane (SF) is a natural compound present in broccoli and other cruciferous vegetables with high concentration. Kallifatidis et al. showed that SF increased the effectiveness of cytotoxic drugs (gemcitabine, cisplatin, 5-fluorouracil, and doxorubicin) against pancreatic cancer stem cells (CSCs) without inducing additional toxicity in mice [16]. Combination of SF with cytotoxic drugs intensified inhibition of spheroid formation, clonogenicity, and aldehyde dehydrogenase 1 activity along with the expression of c-Rel and Notch-1, which indicated that the characteristics of CSCs were targeted. Dimethylamino parthenolide (DMAPT) is a sesquiterpene lactone extracted from the medicinal herb feverfew (Tanacetum parthenium). In association with the suppression of NF- $\kappa \mathrm{B}$, Holcomb et al. indicated that DMAPT enhanced the antiproliferative effects of gemcitabine in pancreatic cancer cells in vitro and in vivo, which supported the evaluation of NF- $\kappa \mathrm{B}-$ targeted agents to complement gemcitabine-based therapies [17]. Dimethylaminoparthenolide (DMAPT) is a novel orally bioavailable analog of parthenolide, a sesquiterpene lactone extracted from the medicinal herb feverfew (Tanacetum parthenium). Yip-Schneider et al. showed that the combination of DMAPT and gemcitabine significantly decreased the multiplicity and incidence of pancreatic adenocarcinomas by reducing the levels of eotaxin, tumor necrosis factoralpha $(\mathrm{TNF}-\alpha)$, macrophage inflammatory protein-1 beta (MIP-1 $\beta$ ), inflammatory cytokines IL-12p40, and monocyte chemotactic protein-1 (MCP-1), all of which are NF- $\kappa$ B target genes [18]. Yip-Schneider et al. also indicated DMAPT and sulindac (nonselective COX inhibitor) in combination with gemcitabine may prevent or delay the progression of premalignant pancreatic lesions in the LSL-Kras (G12D), Pdx-1-Cre mouse model of pancreatic cancer [55]. The phorbol ester, 12-O-tetradecanoylphorbol-13-acetate (TPA), is a major active ingredient extracted from the seed oil of Croton tiglium L., a leafy shrub of the Euphorbiaceae family which is native to Southeastern Asia. Our laboratory indicated that the combination of TPA with gemcitabine synergistically inhibited growth and induced apoptosis in human pancreatic cancer PANC-1 cells cultured in vitro or PANC-1 cells grown in NCr immunodeficient nude mice [19]. $0.16 \mathrm{nM}$ TPA in combination with $0.5 \mu \mathrm{M}$ gemcitabine induced a remarkable increase in the expression of phosphorylated c-Jun NH2-terminal kinase (JNK) in PANC1 cells. Guggulsterone (4,17[20]-pregnadiene-3,16-dione) is a dietary plant sterone obtained from the gum resin of the Indian Ayurvedic medicinal plant, Commiphora mukul, which has been used as traditional medicine since 600 BC. It has been known to show hypolipidemic activity, cardiovascular protecting activity, anti-inflammatory activity, and the activity of antagonist to bile acid receptor (farnesoid $\mathrm{X}$ receptor) [56]. Ahn et al. indicated that guggulsterone combined with gemcitabine augmented growth inhibition of pancreatic cancer cells in vitro and in vivo through downregulation of nuclear factor- $\kappa \mathrm{B}$ activity, suppression of BcL-2 and Akt expression, and activation of Bax and c-Jun $\mathrm{NH}(2)$-terminal kinase [20]. Akasaka et al. indicated that monogalactosyl diacylglycerol (MGDG), a glycoglycerolipid isolated from spinach, combined with gemcitabine revealed synergistic effects of inhibitive proliferation on human pancreatic cancer cell lines BxPC-3, MiaPaCa2, and PANC-1 through the inhibition of DNA replicative pols alpha and gamma activities, compared with MGDG or GEM alone [21]. Glaucarubinone, a quassinoid natural product, was first extracted from the seeds of Simarouba glauca and numerous other species in the family Simaroubaceae. Yeo et 
al. indicated glaucarubinone and gemcitabine synergistically reduced the growth of pancreatic cancer cells in vitro and in vivo via downregulation of P21-activated serine/threonine kinases PAK1 and PAK4 [22]. Thymoquinone is a bioactive constituent isolated from the volatile oil of the black seed (Nigella sativa). Mu et al. showed thymoquinone pretreatment following gemcitabine treatment synergistically caused an increase of apoptosis and tumor growth inhibition in pancreatic cancer cells both in vitro and in vivo, through abrogation of Notchl, PI3K/Akt/mTOR regulated signaling pathways [23]. Namba et al. indicated zidovudine, developed from spongothymidine extracted from Cryptotethya crypta, resensitized gemcitabine-resistant pancreatic cancer cells to gemcitabine by inhibiting the Akt-GSK3 $\beta$-Snail pathway [24]. Piperlongumine is a natural alkaloid/amide component extracted from the fruit of the pepper Piper longum. Wang et al. indicated that Piperlongumine enhanced the antitumor properties of gemcitabine in human pancreatic cancer cells in vitro and in vivo by modulating the NF-kappaB pathway [25].

Escin is a natural mixture of pentacyclic triterpene saponins extracted from the horse chestnut (Aesculus hippocastanum/Aesculus wilsonii Rehd.) seeds that has been used as an antipyretic and an analgesic agent in China. It possesses anticancer activity by induction of growth inhibition and apoptosis in many human cancer cells. Wang et al. showed escin could potentiate the efficacy of gemcitabine against human pancreatic cancer in vitro and in vivo via inactivation of NF- $\kappa \mathrm{B}$ and consequent inhibition of $\mathrm{c}-\mathrm{Myc}, \mathrm{Bcl}-2, \mathrm{Bcl}-\mathrm{xL}$, survivin, COX-2, cyclin D1, and the activation of caspase- 3 [26]. Rimmon et al. indicated that the combination of escin with gemcitabine showed only additive effect in pancreatic cancer PANC-1 cells, while escin combined with cisplatin led to a significant synergistic cytotoxic effect in PANC1 cells by downregulating $\mathrm{NF}-\kappa \mathrm{B}$ signaling pathway [27]. Gum mastic, a natural resin isolated from the leaves and stem of Pistacia lentiscus trees, has been extensively used as both an herbal remedy and a dietary supplement for centuries in Mediterranean and Middle Eastern countries. Huang et al. indicated gum mastic significantly potentiated antiproliferative and apoptotic effects of gemcitabine in both pancreatic cancer BxPC-3 and COLO 357 cells by increasing the expression of IkappaBalpha and Bax protein, blocking NF-kappaB activation and downregulating the expression of $\mathrm{Bcl}-2$ protein [28]. Zyflamend is a polyherbal formulation comprised of ten standardized and concentrated herbal extracts (ginger, holy basil, rosemary, huzhang, oregano, turmeric, Chinese goldthread, baikal skullcap, barberry, and green tea). Kunnumakkara et al. showed Zyflamend in combination with gemcitabine synergically inhibited the growth of human pancreatic cells in vitro and in vivo by inhibiting NF$\kappa \mathrm{B}$ signaling pathways [29]. MK615, called Ume in Japanese, is a sticky extract from Japanese apricot, which has been used to treat intestinal disorders for many years as an antipyretic and an anti-inflammatory agent [57]. Hattori et al. indicated MK615, in both the presence and absence of gemcitabine, significantly inhibited the growth of human pancreatic cancer cells in vitro and in vivo without apparent adverse effects through a reactive oxygen species-dependent mechanism [30]. Herbal preparation of Pao Pereira, obtained from a rainforest tree in the family of Apocynaceae, has long been used by practitioners and oncologic patients in complementary and alternative medicine. Yu et al. showed the combination of the extract of Pao Pereira and gemcitabine had a synergistic effect in inhibiting growth and inducing apoptosis of pancreatic cancer cells [31]. Qingyihuaji formula (QYHJ), consisting of traditional Chinese herbs, has been used for integrative treatment of human pancreatic cancer in China for many years. Xu et al. indicated that QYHJ could enhance the antitumor activity of gemcitabine pancreatic cancer cells by downregulating the expression of Jagged-1 and Notch-4 in Notch signaling pathway [32]. Pan et al. indicated that PBI-05204, a modified supercritical $\mathrm{CO}_{2}$ extract of Nerium oleander, markedly enhanced the antitumor efficacy of gemcitabine in a human pancreatic cancer PANC-1 orthotopic model and human pancreatic cancer cell lines, through downregulation of PI3k/Akt and mTOR pathways [33].

Devil's club Oplopanax horridus (DC), an important medicinal herb of the Pacific Northwest, is a deciduous shrub related in taxonomy to the well-known medicinal plants such as Asian ginseng (Panax ginseng C. A. Meyer), American ginseng (Panax quinquefolius L.), and eleuthero (Eleutherococcus senticosus Maxim. or Acanthopanax senticosus, formerly known as Siberian ginseng). The inner root and stem bark extract of DC show antiproliferation activity on multiple cancer cells in vitro. Tai et al. indicated that there was a significant antiproliferation activity of DC extract alone or in combination with chemotherapeutic agents (gemcitabine, cisplatin, and paclitaxel) on human pancreatic cancer PANC$13 \mathrm{D}$ spheroids and 2D monolayer cells [34]. Cheung et al. also indicated that DC $70 \%$ ethanol extract alone or in combination with chemotherapeutic agents (gemcitabine, cisplatin, and paclitaxel) displayed the high antiproliferation potency on pancreatic endocrine HP62 cells and pancreatic ductal carcinoma BxPC-3 and PANC-1 cells [35]. Summary of pharmacological studies of combination of natural products with gemcitabine was shown in Table 1.

\section{Combination of Natural Products with Other Chemotherapeutic Agents}

Our laboratory indicated that the combination of 12-Otetradecanoylphorbol-13-acetate (TPA) with All-trans Retinoic Acid (ATRA) synergistically inhibited growth and increased apoptosis in human pancreas cancer cells cultured in vitro or pancreas tumor xenografts in immunodeficient mice [36]. The combination of TPA and ATRA induced a remarkable decrease ratio of the percentage of mitotic cells to the percentage of caspase-3-positive cells in the tumors compared with tumors from the vehicle-treated control animals. Our laboratory showed that the combination of TPA and diethyldithiocarbamate (DDTC) synergistically inhibited growth and increased apoptosis in human pancreas cancer cells cultured in vitro and pancreas tumor xenografts in nude mice [37]. The combination of TPA and DDTC induced a remarkable inhibition on the activation of nuclear factor $-\kappa \mathrm{B}(\mathrm{NF}-\kappa \mathrm{B})$ and decreased the expression of $\mathrm{Bcl}-2$. Parthenolide is a sesquiterpene lactone extracted from the medicinal herb feverfew (Tanacetum parthenium). 


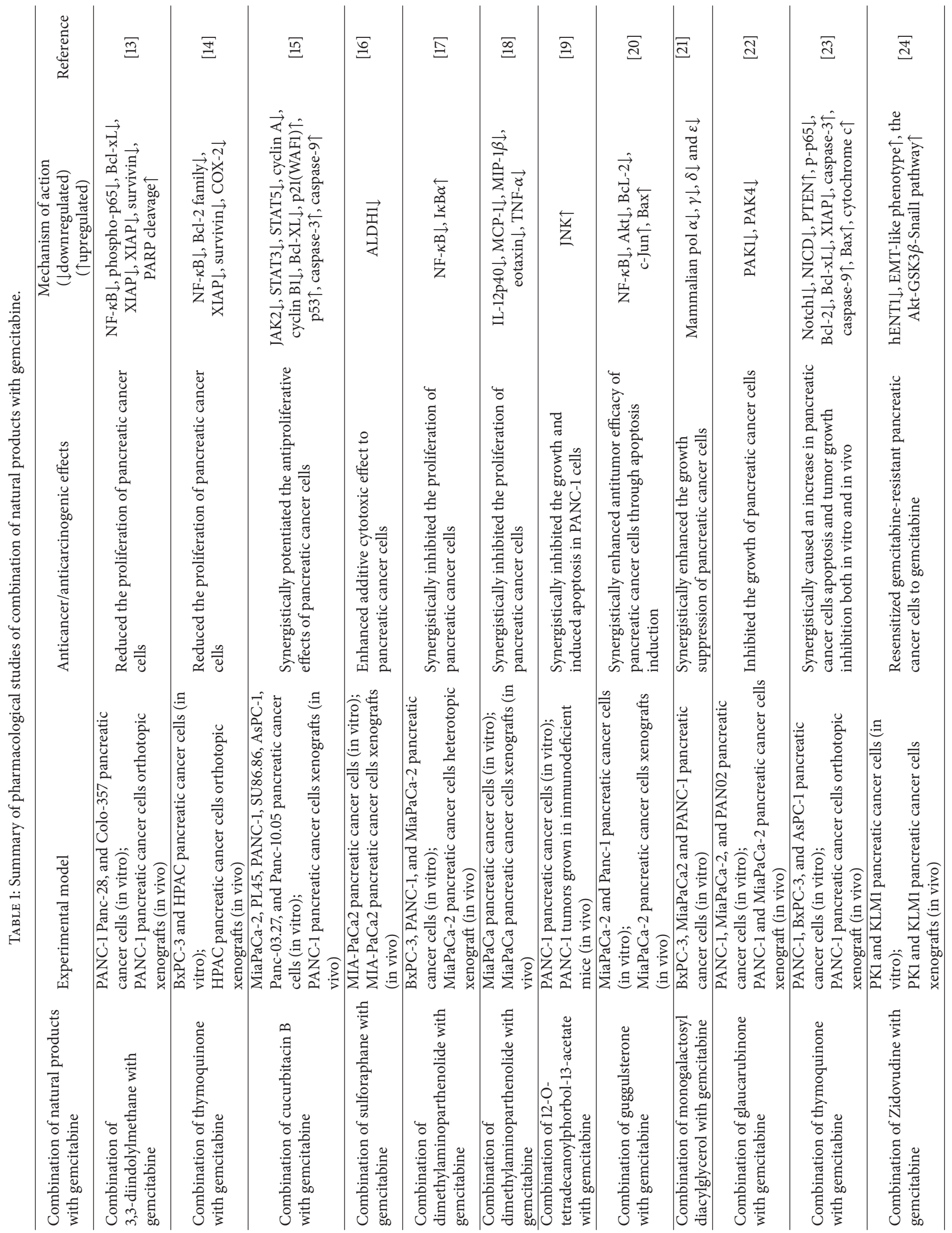




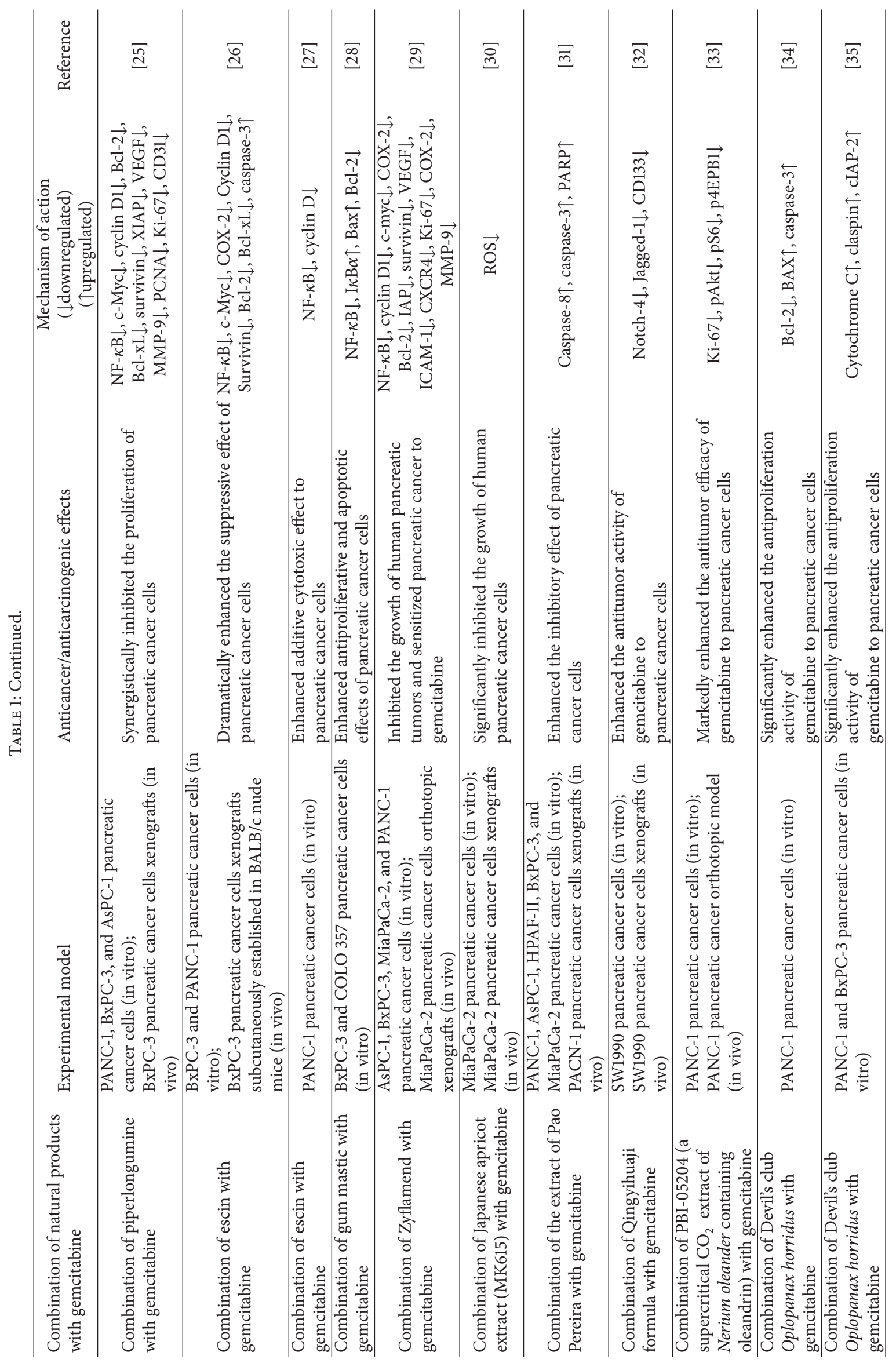


Yip-Schneider et al. showed that the combination of nonsteroidal anti-inflammatory drug sulindac with parthenolide inhibited cell growth additively in pancreatic cancer PANC1 cells and synergistically in pancreatic cancer BxPC-3 and MiaPaCa-2 cells, by increasing the expression level of IkappaBalpha protein and decreasing the binding and transcriptional activities of NF-kappaB DNA [38]. Yip-Schneider et al. also indicated that the combination of the cyclooxygenase 2 inhibitor celecoxib with dimethylaminoparthenolide exhibited significant inhibitory effect of tumor invasion into adjacent organs and metastasis in a carcinogen-induced pancreatic cancer model of Syrian golden hamsters, by decreasing the activity of nuclear factor-kappaB and the expression of prostaglandin E2 and prostaglandin E2 metabolite [39]. Yang et al. indicated that triptolide in combination with hydroxycamptothecin displayed the synergistic cytotoxic effect on pancreatic cancer PANC-1 cells [40]. Sulforaphane, derived from glucoraphanin, was a major glucosinolate and a dietary component in broccoli and broccoli sprouts. $\mathrm{Li}$ et al. indicated that the combination of sulforaphane with Hsp90 inhibitor 17-allylamino-17-demethoxygeldanamycin synergistically inhibited cell growth in vitro and in pancreatic cancer xenograft model in vivo, by decreasing the function of Hsp90 and increasing the activity of caspase-3 [41]. Yuan et al. indicated that the combination of small-molecule BRD4770 (an histone methyltransferase G9a inhibitor) with gossypol (a natural product isolated from cottonseeds) synergistically enhanced the cytotoxicity of p53-mutant pancreatic cancer PANC-1 cells but there was no effect in immortalized nontumorigenic pancreatic cells [42]. The combination of gossypol and BRD4770 induced autophagy-related cell death in pancreatic cancer cells by increasing the levels of LC3-II and the number of autophagosome.

SPES contains 15 herbs: Cervus nippon, Pyrola rotundifolia, Panax ginseng, Ganoderma japonicum, Agrimonia pilosa, Cistanche deserticola, Corydalis bulbosa, Pollen, Glycyrrhiza glabra, Stephania delavayi, Lycoris radiata, Stephania sinica, Zanthoxylum nitidum, Rabdosia rubescens, and Patrinia heterophylla. PC-SPES consists of powders derived from 8 herbs: Dendranthema morifolium, Isatis indigotica, Panax ginseng, Ganoderma lucidum, Serenoa repens, Scutellaria baicalensis, Rabdosia rubescens, and Glycyrrhiza glabra. Schwarz et al. evaluated the antitumor effects of Chinese herbs (SPES and PC-SPES) combined with the chemotherapeutic drugs (doxorubicin or gemcitabine) on eight human pancreatic cancer cell lines (CFPAC, BxPC, MIA, HS-766T, PANC-1, HTB-147, CaPan-2, and ASPC) in vitro [43]. Mediated via induction of apoptosis, both SPES and PC-SPES exhibited significant inhibitory effect on pancreatic cancer cells. Combination effects with either extract appeared to be additive to mildly synergistic in the case of gemcitabine while they appeared to be antagonistic in the case of doxorubicin. Berberis vulgaris, which belongs to Berberidaceae family, is a plant growing in Asia and Europe. Berberis extract has been used for a long time in folk medicine, whose therapeutic value is attributed to the fruits, leaves, bark, and root. Berberine is the most potent of these alkaloids extracted from Berberis vulgaris, responsible for the majority of pleiotropic effects against a number of cancer cell lines. Issat et al. showed cholesterol-reducing agents lovastatin in combination with berberine exert potentiated cytotoxic and/or cytostatic effects against murine Panc 02 pancreatic cancer cells and human MDA-MB231 breast cancer cells [58]. Cell cycle was inhibited in G1 phase after $48 \mathrm{~h}$ of incubation with lovastatin and berberine. The combination of lovastatin and berberine slightly, but significantly, decreased tumor growth in a Panc 02 pancreatic cancer model of mice. Moringa oleifera Lam. (Moringaceae) is a tree that grows widely in the tropics and subtropics of Asia and Africa. Leaves of Moringa oleifera consist of flavonoid pigments, such as kaempferitrin, kaempferol, isoquercitrin, and rhamnetin. Berkovich et al. indicated that Moringa oleifera leaf extract synergistically inhibited tumor growth and enhanced the cytotoxic effect of cisplatin in human pancreas cancer PANC1 cells in vitro, by elevating the sub-G1 cell population of cell cycle and reducing the expression of $\mathrm{p} 65, \mathrm{p}-\mathrm{IkB} \alpha$, and $\operatorname{IkB} \alpha$ proteins [44]. Reis et al. indicated that the combination of lathyranes, the chemical compound isolated from Euphorbia piscatoria, and doxorubicin synergistically enhanced the antiproliferative activity on human pancreatic cells in vitro [59]. Summary of pharmacological studies of combination of natural products with other chemotherapeutic agents was shown in Table 2.

\section{Combination between Natural Products}

Mohammad et al. showed that the combination of (-)gossypol (a natural polyphenolic compound extracted from cotton seeds) with genistein (a prominent soy isoflavone) more significantly inhibited the growth of BxPC-3 pancreatic cancer cells, compared with either agent alone. Genistein, which inactivated NF-kB and caused transcriptional inactivation of $\mathrm{Bcl}-\mathrm{XL}$ and $\mathrm{Bcl}-2$, could be combined with (-)gossypol to inactivate the function of Bcl-XL and Bcl-2 and then enhanced the death of pancreatic cancer cells [45]. Srivastava et al. indicated that sulforaphane, an active compound in cruciferous vegetables, synergistically inhibits self-renewal capacity of pancreatic cancer stem cells with quercetin, a major polyphenol and flavonoid commonly detected in many fruits and vegetables, by inhibiting the expression of Nanog, phosphorylation of FKHR, Bcl-2, XIAP, activating caspase-3, and proteins involved in the epithelialmesenchymal transition (beta-catenin, twist-1, ZEB1, and vimentin) [46]. Ding et al. showed the combination of that wogonin, a naturally occurring flavone, with the structurally related natural flavones apigenin and chrysin could enhance TRAIL-mediated apoptosis in pancreatic carcinoma CaPan-1 cells, by upregulation of TRAIL receptor 2 (TRAIL-R2) and transcriptional downregulation of c-FLIP (a key inhibitor of death receptor signaling) [47]. Yue et al. indicated that metformin combined with aspirin synergistically inhibited tumor growth, migration, and colony formation in human pancreas cancer PANC-1 and BxPC-3 cells cultured in vitro and pancreas tumor xenografts in nude mice, by remarkably inhibiting the phosphorylation of STAT3 and mTOR, significantly downregulating the antiapoptotic proteins $\mathrm{Bcl}-2$ and $\mathrm{Mcl}$ 1 and significantly upregulating the proapoptotic proteins Puma and Bim [48]. Summary of pharmacological studies of combination between natural products was shown in Table 3. 


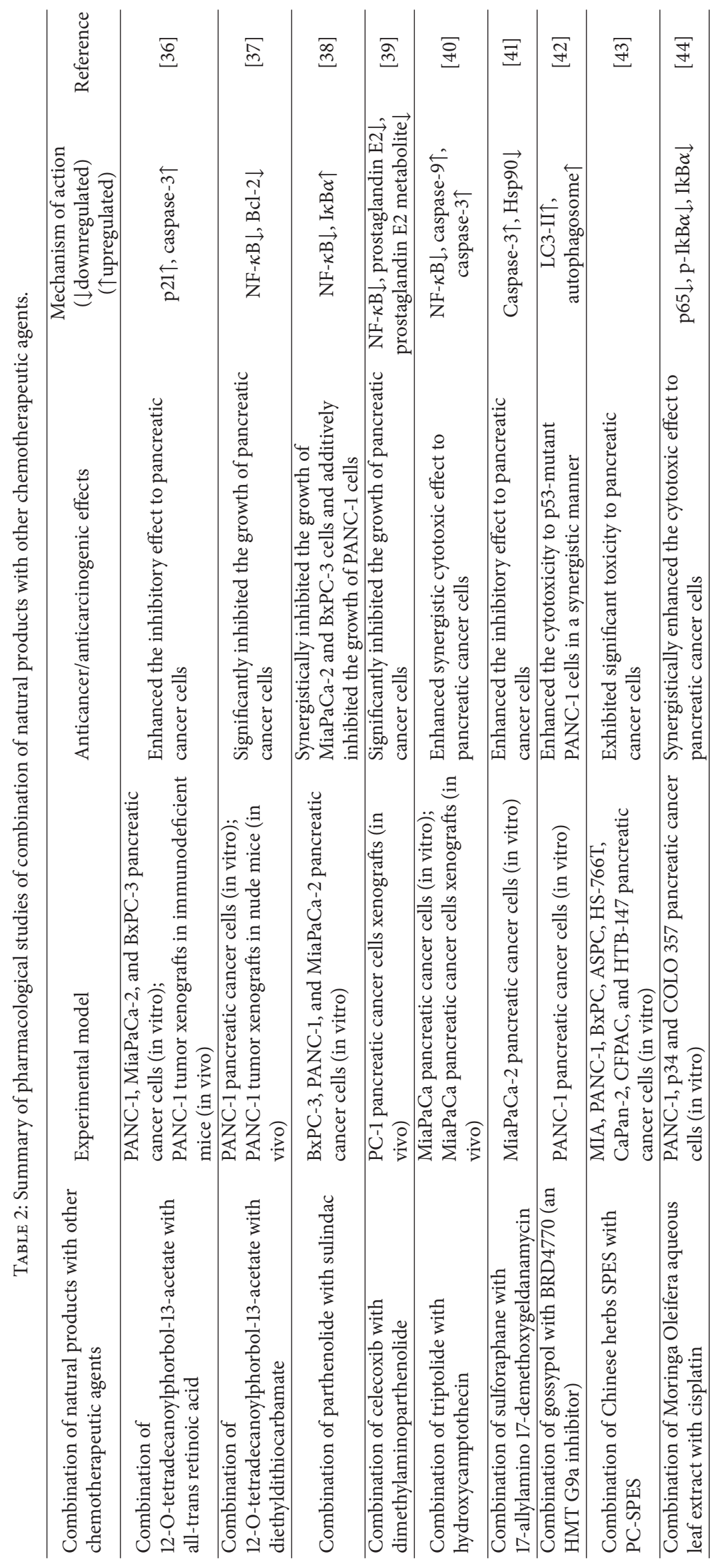




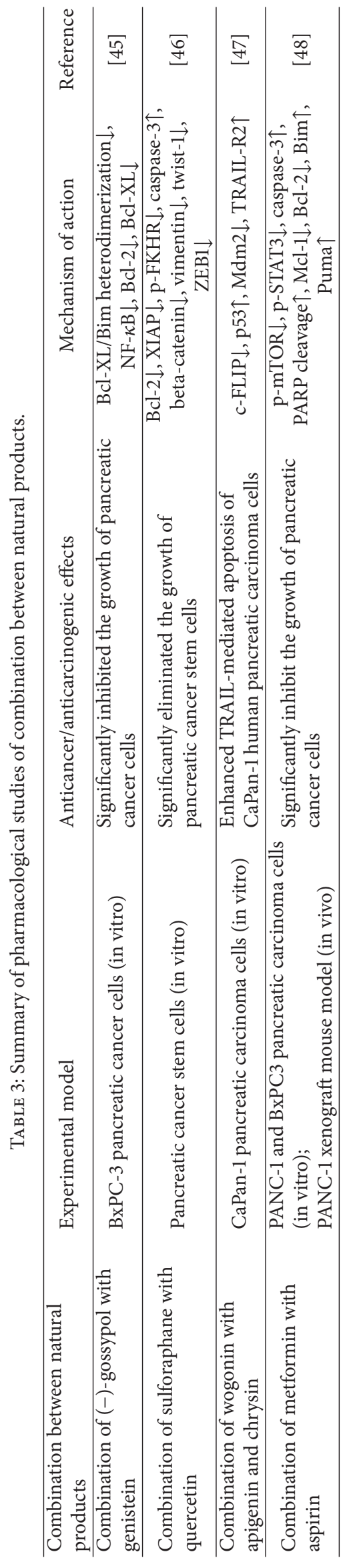




\section{Combination of Natural Products in Early Phases of Clinical Trials}

Hauns et al. indicated that Ginkgo biloba extract GBE 761 ONC combined with 5-fluorouracil (5-FU) was shown favourable effect to treat the patients with pancreatic cancer in the clinical trial phase II study, compared to the clinical trial of 5-FU monotherapy [60]. Curcumin, which is called diferuloyl methane, is a hydrophobic polyphenol isolated from rhizome (turmeric) of the herb Curcuma longa. Curcumin has shown various activities, such as a mediator of chemoresistance and radio-resistance, antioxidant, antiinflammatory, and immunomodulatory activities, enhancing of the apoptotic process, cytokines release, and antiangiogenic properties. The anticancer effect has been seen in a few clinical trials, mainly as a native chemoprevention agent in colon and pancreatic cancer, cervical neoplasia, and Barrett's metaplasia [61]. Although curcumin may potentiate the antitumor effect of gemcitabine by intervening with several intracellular signal transduction pathways in pancreatic cancer cells in vitro and in vivo, Epelbaum et al. showed that a combination of gemcitabine and oral curcumin at a dose of $8,000 \mathrm{mg} /$ day to treat the patients with advanced pancreatic cancer is not feasible and $29 \%$ patients had to stop oral curcumin due to gastrointestinal toxicity [62]. The preliminary results suggest that a combination of curcumin and gemcitabine for the patients with advanced pancreatic cancer is feasible. Mistletoe extracts from the medicinal herb Viscum album L. are widely used to treat cancer patients in Europe. Matthes et al. indicated that mistletoe extract (Iscador ${ }^{\circledR}$, Weleda, Arlesheim, Switzerland) could be supportive care in an adjuvant chemotherapy setting with gemcitabine or 5-fluorouracil (5-FU) in the patients undergoing curative intent resection of pancreatic cancer [63]. Löhr et al. reported the phase Ib study of 16 chemotherapy-native patients with inoperable pancreatic carcinoma treated with gemcitabine and AXP107-11, the sodium salt dihydrate form of genistein (genistein-SSDH, a novel multicomponent crystalline form of genistein) [64]. The results demonstrated that treatment of pancreatic cancer patients with AXP107-11 in combination with gemcitabine led to a favorable pharmacokinetics with high serum levels without toxicity.

Traditional Chinese herbal formulation Huang-Qin-Tang (HQT), which had been documented for almost 1800 years to treat common gastrointestinal distress, such as diarrhea, headache, abdominal spasms, nausea, fever, subcardiac distention, extreme thirst, and vomiting, included four distinct herbs: the roots of Scutellaria baicalensis Georgi. (scute), Glycyrrhiza uralensis Fisch. (licorice), and Paeonia lactiflora Pall. (peony) and the fruit of Ziziphus jujuba Mill. (Chinese date). Each herb of Huang-Qin-Tang had an unusual pharmacological profile, which was involved in antiviral and anticancer activity, liver protection, hematological and immunological modulation, appetite improvement, and analgesic activity. Botanical formulation PHY906, a pharmaceutical grade of Huang-Qin-Tang, was explored as an adjuvant for chemotherapy and targeted therapy of tumors by the teams led by Professor Yung-Chi Cheng in Yale University School of Medicine and PhytoCeutica, Inc. PHY906 was distinguished from Huang-Qin-Tang which is currently available in the market, due to the unique and defined procedures for its characterization, preparation, and quality control. An open label phase I/II study of PHY906 in combination with capecitabine in the patients with advanced pancreatic was concluded at Yale Cancer Center in 2009. Saif et al. conducted phase I study in the patients with advanced pancreatic and gastrointestinal malignancies using PHY906 combined with capecitabine to determine the maximum tolerated dose (MTD) of capecitabine in combination with PHY906 [65]. The results of phase I study indicated that the MTD of capecitabine was $1500 \mathrm{mg} / \mathrm{m}^{2}$ BID administered in a $7 / 7$ schedule, combined with PHY906 $800 \mathrm{mg}$ BID on days 14. Saif et al. also carried out phase II study in the patients with advanced pancreatic cancer who were previously treated with gemcitabine-based regimens to explore the efficacy of capecitabine in combination with PHY906 [66]. The results of phase II study showed that capecitabine combined with PHY906 displayed a feasible and safe salvage therapy after the failure of gemcitabine for advanced pancreatic cancer.

\section{Conclusions and Future Directions}

Although there are great improvements in the treatment of many common cancers in clinic (e.g., prostate cancer and breast cancer) in recent decades, pancreatic cancer still remains the most deadly diagnosed cancer and represents a major challenge [10]. Herbal remedies have been used to treat various diseases for thousands of years in numerous countries, such as China, Egypt, Japan, and Korea, which have come to be accepted as forms of complementary and alternative medicines in western countries [67]. Therefore, natural products/herbal medicines play important roles in prevention and therapy of pancreatic cancer as a promising adjunctive approach. They have been credited with substantial advantages, including improving immune system, suppressing tumor progression, enhancing beneficial effects, and lessening adverse/side effects of chemotherapy and radiotherapy [11]. Unlike western medicine which generally uses purified chemical compounds and targets single physiological endpoints, natural products/herbal medicines usually consist of multiple components and herbs which act/interact simultaneously through various cellular signal mechanisms and molecular targets [10]. These multiple herbs serve various functions; some may improve the efficacy while others may increase the bioavailability or decrease the toxicity. Even isolated compounds from natural products/herbal medicines may display multiple effects, such as improving the efficacy and decreasing the toxicity of chemotherapy and/or radiotherapy. So, there are various natural products/herbal medicines targeting multiple cancerrelated proteins and pathways, which make them an attractive direction as supplementary therapeutics for the patients with pancreatic cancer [11].

Herbal medicine formulations, including Chinese herbal medicine, have been originated from empirical observations in humans over thousands of years. As a complementary and alternative therapy, Chinese herbal medicine has increasingly drawn an interest among international cancer research 
studies because it can increase the efficacy and decrease the toxicity when combined with chemotherapy and/or radiotherapy. Moreover, according to NCCN clinical practice guidelines, the integration of palliative care in cancer patients has become standard oncology practice when a patient is diagnosed with metastatic or advanced cancer, including pancreatic cancer [12]. Chinese herbal medicine has a longstanding history in China and it is deeply embedded in urban and rural populations as a measure of palliative care. The phenomena are attributed to the fact that the origin and the development of traditional Chinese medicine are intricately entwined with Chinese history, economy, politics, and culture, whose compelling efficacy has been attested. Though Chinese herbal medicine has multiple complicated components and probable adverse effects, its application has been widely embraced in clinical practice, especially throughout China. Chinese herbal medicine has also been accepted into Chinese clinical practice guidelines to treat pancreatic cancers [12]. Preclinical studies have demonstrated that Chinese herbal medicine can suppress tumor proliferation and metastasis. Recent reported studies have shown that 90\% Chinese patients with cancer are treated with diverse Chinese herbal medicine alone or in combination with chemotherapy and/or radiotherapy during their treatment regimen [12]. Chinese herbal medicine can increase the effect of antitumor therapies and improve the quality of life or the performance status of pancreatic cancer patients, which will provide more evidence to promote the application of Chinese herbal medicine to benefit the patients with pancreatic cancer in the world.

\section{Competing Interests}

The authors confirmed that this article content has no conflict of interests. The authors declared no potential conflict of interests with respect to the research, authorship, and/or publication of this article.

\section{Authors' Contributions}

Qingxi Yue and Guogang Gao contributed equally to this work.

\section{Acknowledgments}

This work was supported in part by grants from National Nature Science Foundation (81302809 and 81272452), Research Program of Shanghai Municipal Commission of Health and Family Planning (20154Y0149), fund of Shanghai Jiaotong University (syzrc2014-001, syz2014-005, and YG2015MS24), and National Cancer Institute Foundation (P30-CA072720). The authors would like to thank Scientific Creation (Shanghai) Bio-Tech Co. Limited for technology support and helpful advice.

\section{References}

[1] L. A. Torre, F. Bray, R. L. Siegel, J. Ferlay, J. Lortet-Tieulent, and A. Jemal, "Global cancer statistics, 2012," CA: A Cancer Journal for Clinicians, vol. 65, no. 2, pp. 87-108, 2015.
[2] R. L. Siegel, K. D. Miller, and A. Jemal, “Cancer statistics, 2016," CA Cancer Journal for Clinicians, vol. 66, no. 1, pp. 7-30, 2016.

[3] W. Chen, R. Zheng, P. D. Baade et al., "Cancer statistics in China, 2015," CA Cancer Journal for Clinicians, vol. 66, no. 2, pp. 115132, 2016.

[4] A. S. Paulson, H. S. Tran Cao, M. A. Tempero, and A. M. Lowy, "Therapeutic advances in pancreatic cancer," Gastroenterology, vol. 144, no. 6, pp. 1316-1326, 2013.

[5] L. Perkhofer, T. J. Ettrich, and T. Seufferlein, "Pancreatic cancer: progress in systemic therapy," Gastrointestinal Tumors, vol. 1, no. 4, pp. 167-179, 2014.

[6] S. D. Stan, S. V. Singh, and R. E. Brand, "Chemoprevention strategies for pancreatic cancer," Nature Reviews Gastroenterology and Hepatology, vol. 7, no. 6, pp. 347-356, 2010.

[7] J.-E. Ying, L.-M. Zhu, and B.-X. Liu, "Developments in metastatic pancreatic cancer: is gemcitabine still the standard?" World Journal of Gastroenterology, vol. 18, no. 8, pp. 736-745, 2012.

[8] A. Akinleye, C. Iragavarapu, M. Furqan, S. Cang, and D. Liu, "Novel agents for advanced pancreatic cancer," Oncotarget, vol. 6, no. 37, pp. 39521-39537, 2015.

[9] S. Perwez Hussain, "Pancreatic cancer: current progress and future challenges," International Journal of Biological Sciences, vol. 12, no. 3, pp. 270-272, 2016.

[10] A. Mohammed, N. B. Janakiram, S. Pant, and C. V. Rao, "Molecular targeted intervention for pancreatic cancer," Cancers, vol. 7, no. 3, pp. 1499-1542, 2015.

[11] L. Li and P. S. Leung, "Use of herbal medicines and natural products: an alternative approach to overcoming the apoptotic resistance of pancreatic cancer," International Journal of Biochemistry and Cell Biology, vol. 53, pp. 224-236, 2014.

[12] B. Li, R. Gan, Q. Yang et al., "Chinese herbal medicines as an adjunctive therapy for unresectable pancreatic cancer: a systematic review and meta-analysis," Evidence-Based Complementary and Alternative Medicine, vol. 2015, Article ID 350730, 15 pages, 2015.

[13] S. Banerjee, Z. Wang, D. Kong, and F. H. Sarkar, "3,3" Diindolylmethane enhances chemosensitivity of multiple chemotherapeutic agents in pancreatic cancer," Cancer Research, vol. 69, no. 13, pp. 5592-5600, 2009.

[14] S. Banerjee, A. O. Kaseb, Z. Wang et al., "Antitumor activity of gemcitabine and oxaliplatin is augmented by thymoquinone in pancreatic cancer," Cancer Research, vol. 69, no. 13, pp. 55755583,2009 .

[15] N. H. Thoennissen, G. B. Iwanski, N. B. Doan et al., "Cucurbitacin $\mathrm{B}$ induces apoptosis by inhibition of the JAK/STAT pathway and potentiates antiproliferative effects of gemcitabine on pancreatic cancer cells," Cancer Research, vol. 69, no. 14, pp. 5876-5884, 2009.

[16] G. Kallifatidis, S. Labsch, V. Rausch et al., "Sulforaphane increases drug-mediated cytotoxicity toward cancer stem-like cells of pancreas and prostate," Molecular Therapy, vol. 19, no. 1, pp. 188-195, 2011.

[17] B. K. Holcomb, M. T. Yip-Schneider, J. A. Waters, J. D. Beane, P. A. Crooks, and C. M. Schmidt, "Dimethylamino parthenolide enhances the inhibitory effects of gemcitabine in human pancreatic cancer cells," Journal of Gastrointestinal Surgery, vol. 16, no. 7, pp. 1333-1340, 2012.

[18] M. T. Yip-Schneider, H. Wu, K. Stantz, N. Agaram, P. A. Crooks, and C. M. Schmidt, "Dimethylaminoparthenolide and gemcitabine: a survival study using a genetically engineered 
mouse model of pancreatic cancer," BMC Cancer, vol. 13, article no. $194,2013$.

[19] X. Zheng, X.-X. Cui, Z. Gao et al., "Effects of 12-Otetradecanoylphorbol-13-acetate in combination with gemcitabine on Panc-1 pancreatic cancer cells cultured in vitro or Panc-1 tumors grown in immunodeficient mice," International Journal of Oncology, vol. 41, no. 6, pp. 2269-2275, 2012.

[20] D.-W. Ahn, J. K. Seo, S. H. Lee et al., "Enhanced antitumor effect of combination therapy with gemcitabine and guggulsterone in pancreatic cancer," Pancreas, vol. 41, no. 7, pp. 1048-1057, 2012.

[21] H. Akasaka, R. Sasaki, K. Yoshida et al., "Monogalactosyl diacylglycerol, a replicative DNA polymerase inhibitor, from spinach enhances the anti-cell proliferation effect of gemcitabine in human pancreatic cancer cells," Biochimica et Biophysica Acta, vol. 1830, no. 3, pp. 2517-2525, 2013.

[22] D. Yeo, N. Huynh, J. A. Beutler et al., "Glaucarubinone and gemcitabine synergistically reduce pancreatic cancer growth via down-regulation of P21-activated kinases," Cancer Letters, vol. 346, no. 2, pp. 264-272, 2014.

[23] G.-G. Mu, L.-L. Zhang, H.-Y. Li, Y. Liao, and H.-G. Yu, "Thymoquinone pretreatment overcomes the insensitivity and potentiates the antitumor effect of gemcitabine through abrogation of notchl, PI3K/Akt/mTOR regulated signaling pathways in pancreatic cancer," Digestive Diseases and Sciences, vol. 60, no. 4, pp. 1067-1080, 2015.

[24] T. Namba, R. Kodama, S. Moritomo, T. Hoshino, and T. Mizushima, "Zidovudine, an anti-viral drug, resensitizes gemcitabine-resistant pancreatic cancer cells to gemcitabine by inhibition of the Akt-GSK3 $\beta$-Snail pathway," Cell Death and Disease, vol. 6, no. 6, Article ID e1795, 2015.

[25] Y. Wang, X. Wu, Y. Zhou, H. Jiang, S. Pan, and B. Sun, "Piperlongumine suppresses growth and sensitizes pancreatic tumors to gemcitabine in a xenograft mouse model by modulating the NF-kappa B pathway," Cancer Prevention Research, vol. 9, no. 3, pp. 234-244, 2016.

[26] Y.-W. Wang, S.-J. Wang, Y.-N. Zhou, S.-H. Pan, and B. Sun, "Escin augments the efficacy of gemcitabine through downregulation of nuclear factor- $\kappa \mathrm{B}$ and nuclear factor- $\kappa \mathrm{B}$-regulated gene products in pancreatic cancer both in vitro and in vivo," Journal of Cancer Research and Clinical Oncology, vol. 138, no. 5, pp. 785-797, 2012.

[27] A. Rimmon, A. Vexler, L. Berkovich, G. Earon, I. Ron, and S. Lev-Ari, "Escin chemosensitizes human pancreatic cancer cells and inhibits the nuclear factor-kappaB signaling pathway," Biochemistry Research International, vol. 2013, Article ID 251752, 9 pages, 2013.

[28] X.-Y. Huang, H.-C. Wang, Z. Yuan et al., "Gemcitabine combined with gum mastic causes potent growth inhibition and apoptosis of pancreatic cancer cells," Acta Pharmacologica Sinica, vol. 31, no. 6, pp. 741-745, 2010.

[29] A. B. Kunnumakkara, B. Sung, J. Ravindran et al., "Zyflamend suppresses growth and sensitizes human pancreatic tumors to gemcitabine in an orthotopic mouse model through modulation of multiple targets," International Journal of Cancer, vol. 131, no. 3, pp. E292-E303, 2012.

[30] M. Hattori, K. Kawakami, M. Akimoto, K. Takenaga, J. Suzumiya, and Y. Honma, "Antitumor effect of Japanese apricot extract (MK615) on human cancer cells in vitro and in vivo through a reactive oxygen species-dependent mechanism," Tumori, vol. 99, no. 2, pp. 239-248, 2013.
[31] J. Yu, J. Drisko, and Q. Chen, "Inhibition of pancreatic cancer and potentiation of gemcitabine effects by the extract of Pao Pereira," Oncology Reports, vol. 30, no. 1, pp. 149-156, 2013.

[32] Y. Xu, F. Zhu, S. Xu, and L. Liu, "Anti-tumor effect of the extract from Qingyihuaji formula on pancreatic cancer by down-regulating Notch-4 and Jagged-1," Journal of Traditional Chinese Medicine, vol. 35, no. 1, pp. 77-83, 2015.

[33] Y. Pan, P. Rhea, L. Tan et al., "PBI-05204, a supercritical $\mathrm{CO}_{2}$ extract of Nerium oleander, inhibits growth of human pancreatic cancer via targeting the PI3K/mTOR pathway," Investigational New Drugs, vol. 33, no. 2, pp. 271-279, 2015.

[34] J. Tai, S. S. C. Cheung, D. Ou, G. L. Warnock, and D. Hasman, "Antiproliferation activity of Devil's club (Oplopanax horridus) and anticancer agents on human pancreatic cancer multicellular spheroids," Phytomedicine, vol. 21, no. 4, pp. 506-514, 2014.

[35] S. S. C. Cheung, J. Tai, D. Hasman, D. Ou, and G. L. Warnock, "Inhibition of human pancreatic cancer cell proliferation by Devil's club oplopanax horridus and its polyacetylene bioactive compound," Nutrition and Cancer, vol. 67, no. 6, pp. 954-964, 2015.

[36] G. E. Avila, X. Zheng, X. X. Cui et al., "Inhibitory effects of 12-O-tetradecanoylphorbol-13-acetate alone or in combination with all-trans retinoic acid on the growth of cultured human pancreas cancer cells and pancreas tumor xenografts in immunodeficient mice," Journal of Pharmacology and Experimental Therapeutics, vol. 315, no. 1, pp. 170-187, 2005.

[37] H. Huang, K. Cao, S. Malik et al., "Combination of 12-Otetradecanoylphorbol-13-Acetate with diethyldithiocarbamate markedly inhibits pancreatic cancer cell growth in 3D culture and in immunodeficient mice," International Journal of Molecular Medicine, vol. 35, no. 6, pp. 1617-1624, 2015.

[38] M. T. Yip-Schneider, H. Nakshatri, C. J. Sweeney, M. S. Marshall, E. A. Wiebke, and C. M. Schmidt, "Parthenolide and sulindac cooperate to mediate growth suppression and inhibit the nuclear factor- $\kappa \mathrm{B}$ pathway in pancreatic carcinoma cells," Molecular Cancer Therapeutics, vol. 4, no. 4, pp. 587-594, 2005.

[39] M. T. Yip-Schneider, H. Wu, V. Njoku et al., "Effect of celecoxib and the novel anti-cancer agent, dimethylamino- parthenolide, in a developmental model of pancreatic cancer," Pancreas, vol. 37, no. 3, pp. e45-e53, 2008.

[40] S.-W. Yang, W. Wang, X.-Y. Xie, W.-P. Zhu, and F.-Q. Li, “In vitro synergistic cytotoxic effect of triptolide combined with hydroxycamptothecin on pancreatic cancer cells," American Journal of Chinese Medicine, vol. 39, no. 1, pp. 121-134, 2011.

[41] Y. Li, T. Zhang, S. J. Schwartz, and D. Sun, "Sulforaphane potentiates the efficacy of 17-allylamino 17- demethoxygeldanamycin against pancreatic cancer through enhanced abrogation of Hsp90 chaperone function," Nutrition and Cancer, vol. 63, no. 7, pp. 1151-1159, 2011.

[42] Y. Yuan, A. J. Tang, A. B. Castoreno et al., "Gossypol and an HMT G9a inhibitor act in synergy to induce cell death in pancreatic cancer cells," Cell Death \& Disease, vol. 4, article no. e690, 2013.

[43] R. E. Schwarz, C. A. Donohue, D. Sadava, and S. E. Kane, "Pancreatic cancer in vitro toxicity mediated by Chinese herbs SPES and PC-SPES: implications for monotherapy and combination treatment," Cancer Letters, vol. 189, no. 1, pp. 59-68, 2003.

[44] L. Berkovich, G. Earon, I. Ron, A. Rimmon, A. Vexler, and S. Lev-Ari, "Moringa Oleifera aqueous leaf extract downregulates nuclear factor-kappaB and increases cytotoxic effect of chemotherapy in pancreatic cancer cells," BMC Complementary and Alternative Medicine, vol. 13, article 212, 2013. 
[45] R. M. Mohammad, S. Wang, S. Banerjee, X. Wu, J. Chen, and F. H. Sarkar, "Nonpeptidic small-molecule inhibitor of Bcl-2 and $\mathrm{Bcl}-\mathrm{X}_{L},(-)$-gossypol, enhances biological effect of genistein against BxPC-3 human pancreatic cancer cell line," Pancreas, vol. 31, no. 4, pp. 317-324, 2005.

[46] R. K. Srivastava, S. N. Tang, W. Zhu, D. Meeker, and S. Shankar, "Sulforaphane synergizes with quercetin to inhibit self-renewal capacity of pancreatic cancer stem cells," Frontiers in Bioscience, vol. 3, pp. 515-528, 2011.

[47] J. Ding, G. Polier, R. Köhler, M. Giaisi, P. H. Krammer, and M. Li-Weber, "Wogonin and related natural flavones overcome tumor necrosis factor-related apoptosis-inducing ligand (TRAIL) protein resistance of tumors by down-regulation of c-FLIP protein and up-regulation of TRAIL receptor 2 expression," The Journal of Biological Chemistry, vol. 287, no. 1, pp. 641649, 2012.

[48] W. Yue, X. Zheng, Y. Lin et al., "Metformin combined with aspirin significantly inhibit pancreatic cancer cell growth in vitro and in vivo by suppressing antiapoptotic proteins Mcl-1 and Bcl-2," Oncotarget, vol. 6, no. 25, pp. 21208-21224, 2015.

[49] T. C. McMorris, M. J. Kelner, W. Wang, J. Yu, L. A. Estes, and R. Taetle, "(Hydroxymethyl)acylfulvene: an illudin derivative with superior antitumor properties," Journal of Natural Products, vol. 59, no. 9, pp. 896-899, 1996.

[50] S. E. Witta, G. S. Eckhardt, M. Rothenberg et al., "A phase I combination trial of irofulven and gemcitabine in patients with advanced solid malignancies," Proc ASCO, vol. 22, article 138, 2003.

[51] E. S. Van Laar, S. Roth, S. Weitman, J. R. MacDonald, and S. J. Waters, "Activity of irofulven against human pancreatic carcinoma cell lines in vitro and in vivo," Anticancer Research, vol. 24, no. 1, pp. 59-65, 2004.

[52] S. M. Kupchan, D. R. Streelman, and A. T. Sneden, "Psorospermin, a new antileukemic xanthone from psorospermum febrifugum," Journal of Natural Products (Lloydia), vol. 43, no. 2, pp. 296-301, 1980.

[53] J. M. Cassady, W. M. Baird, and C.-J. Chang, "Natural products as a source of potential cancer chemotherapeutic and chemopreventive agents," Journal of Natural Products, vol. 53, no. 1, pp. 23-41, 1990.

[54] I. M. Fellows, M. Schwaebe, T. S. Dexheimer et al., "Determination of the importance of the stereochemistry of psorospermin in topoisomerase II-induced alkylation of DNA and in vitro and in vivo biological activity," Molecular Cancer Therapeutics, vol. 4, no. 11, pp. 1729-1739, 2005.

[55] M. T. Yip-Schneider, H. Wu, R. H. Hruban, A. M. Lowy, P. A. Crooks, and C. M. Schmidt, "Efficacy of dimethylaminoparthenolide and sulindac in combination with gemcitabine in a genetically engineered mouse model of pancreatic cancer," Pancreas, vol. 42, no. 1, pp. 160-167, 2013.

[56] C. J. Sinal and F. J. Gonzalez, "Guggulsterone: an old approach to a new problem," Trends in Endocrinology and Metabolism, vol. 13, no. 7, pp. 275-276, 2002.

[57] J. R. Hanson, "The development of strategies for terpenoid structure determination," Natural Product Reports, vol. 18, no. 6, pp. 607-617, 2001.

[58] T. Issat, D. Nowis, J. Bil, M. Winiarska, M. Jakobisiak, and J. Golab, "Antitumor effects of the combination of cholesterol reducing drugs," Oncology Reports, vol. 26, no. 1, pp. 169-176, 2011.

[59] M. A. Reis, A. Paterna, A. Mónico, J. Molnar, H. Lage, and M.-J. U. Ferreira, "Diterpenes from Euphorbia piscatoria: synergistic interaction of lathyranes with doxorubicin on resistant cancer cells," Planta Medica, vol. 80, no. 18, pp. 1739-1745, 2014.

[60] B. Hauns, B. Häring, S. Köhler, K. Mross, P. Robben-Bathe, and C. Unger, "Phase II study with 5-fluorouracil and ginkgo biloba extract (GBE 761 ONC) in patients with pancreatic cancer," Arzneimittel-Forschung, vol. 49, no. 12, pp. 1030-1034, 1999.

[61] G. Bar-Sela, R. Epelbaum, and M. Schaffer, "Curcumin as an anti-cancer agent: review of the gap between basic and clinical applications," Current Medicinal Chemistry, vol. 17, no. 3, pp. 190-197, 2010.

[62] R. Epelbaum, M. Schaffer, B. Vizel, V. Badmaev, and G. BarSela, "Curcumin and gemcitabine in patients with advanced pancreatic cancer," Nutrition and Cancer, vol. 62, no. 8, pp. 11371141, 2010.

[63] H. Matthes, W. E. Friedel, P. R. Bock, and K. S. Zänker, "Molecular mistletoe therapy: friend or foe in established antitumor protocols? a multicenter, controlled, retrospective pharmaco-epidemiological study in pancreas cancer," Current Molecular Medicine, vol. 10, no. 4, pp. 430-439, 2010.

[64] J.-M. Löhr, M. Karimi, B. Omazic et al., "A phase I dose escalation trial of AXP107-11, a novel multi-component crystalline form of genistein, in combination with gemcitabine in chemotherapy-naive patients with unresectable pancreatic cancer," Pancreatology, vol. 16, no. 4, pp. 640-645, 2016.

[65] M. W. Saif, F. Lansigan, S. Ruta et al., "Phase I study of the botanical formulation PHY906 with capecitabine in advanced pancreatic and other gastrointestinal malignancies," Phytomedicine, vol. 17, no. 3-4, pp. 161-169, 2010.

[66] M. W. Saif, J. Li, L. Lamb et al., "First-in-human phase II trial of the botanical formulation PHY906 with capecitabine as second-line therapy in patients with advanced pancreatic cancer," Cancer Chemotherapy and Pharmacology, vol. 73, no. 2, pp. 373-380, 2014.

[67] S. T. Chen, J. Dou, R. Temple, R. Agarwal, K.-M. Wu, and S. Walker, "New therapies from old medicines," Nature Biotechnology, vol. 26, no. 10, pp. 1077-1083, 2008. 


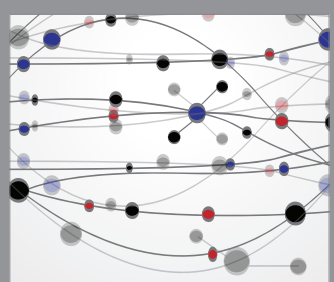

The Scientific World Journal
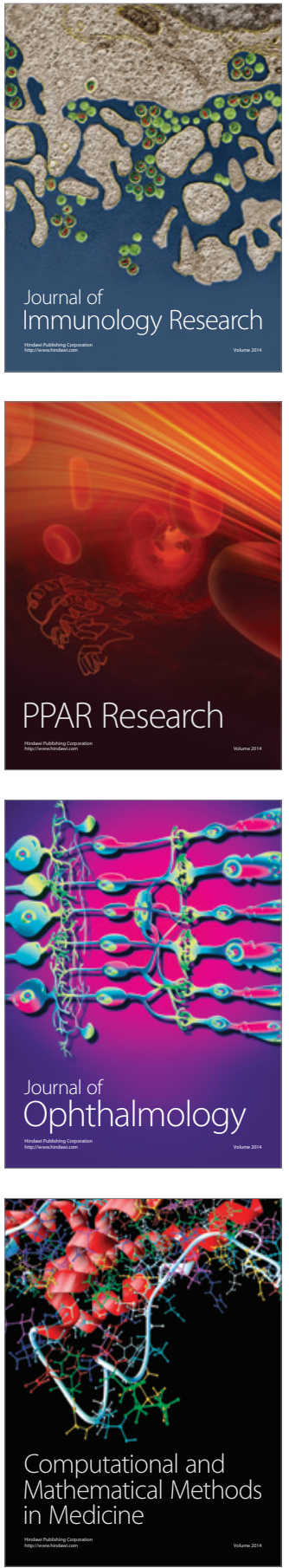

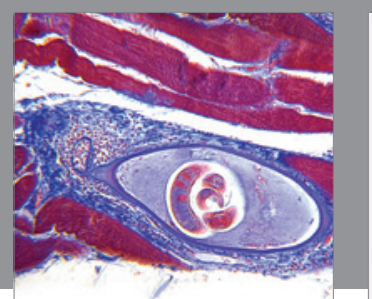

Gastroenterology Research and Practice
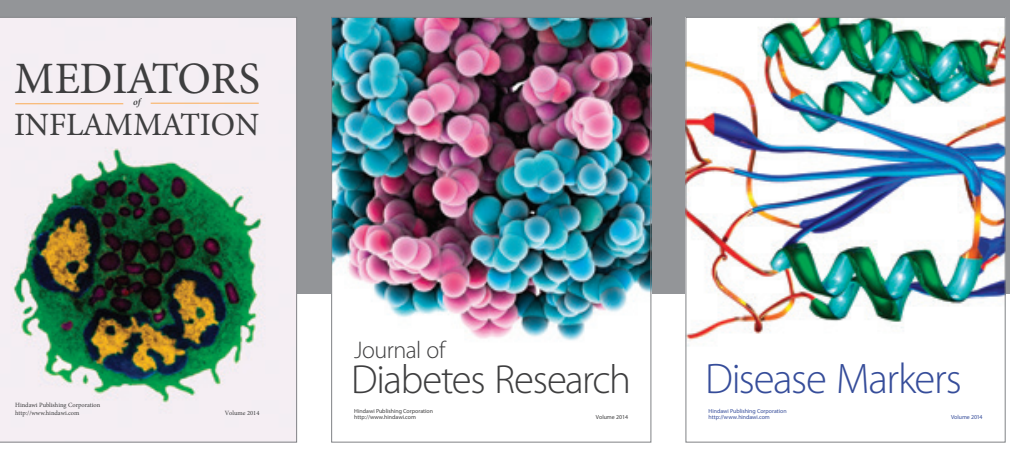

Disease Markers

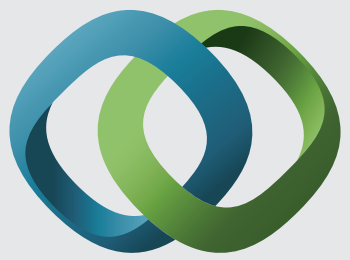

\section{Hindawi}

Submit your manuscripts at

https://www.hindawi.com
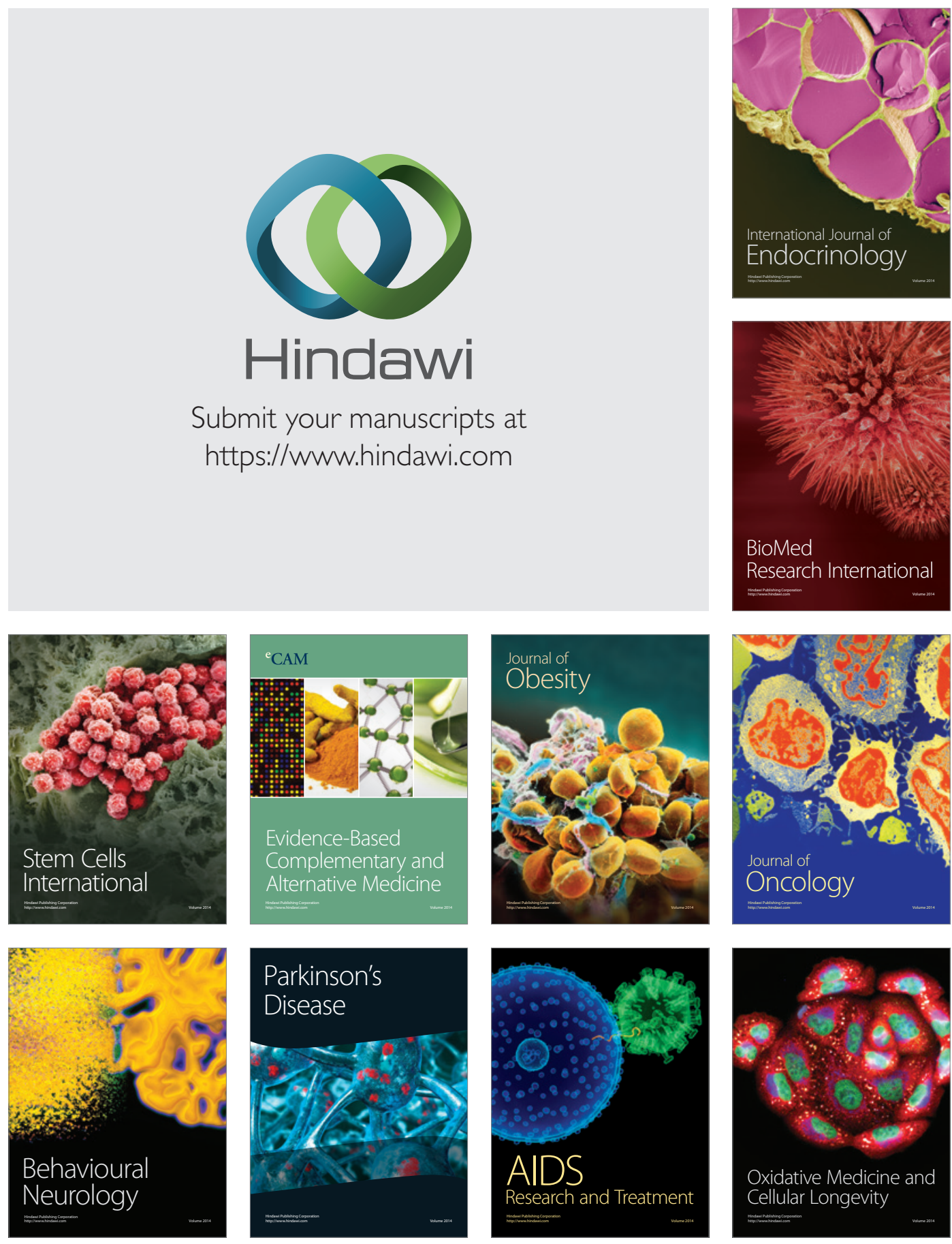